PHYSICAL REVIEW D 95, 094010 (2017)

\title{
Possible small- $x$ effects in the Kaluza-Klein graviton and radion production at high energies
}

\author{
A. V. Lipatov ${ }^{1,2}$ and M. A. Malyshev ${ }^{1}$ \\ ${ }^{1}$ Skobeltsyn Institute of Nuclear Physics, Lomonosov Moscow State University, Moscow 119991, Russia \\ ${ }^{2}$ Joint Institute for Nuclear Research, Dubna 141980, Moscow Region, Russia
}

(Received 11 January 2017; published 19 May 2017)

\begin{abstract}
We study the single graviton production at the CERN LHC using the $k_{T}$-factorization approach of QCD. We consider the Arkani-Hamed-Dimopoulos-Dvali scenario and Randall-Sundrum model with one warped extra dimension and derive the production amplitudes for spin-2 (Kaluza-Klein excitation of the graviton) and spin-0 (radion) states, including subsequent graviton decay into the dilepton or diphoton pairs. We use the transverse momentum dependent (unintegrated) parton densities in a proton obtained from the Ciafaloni-Catani-Fiorani-Marchesini evolution equation, which resums the leading logarithmic small- $x$ corrections to the production cross sections, and estimate the uncertainties of our predictions. We demonstrate that the small- $x$ effects can manifest themselves in the different angular distributions of graviton decay products and give some examples of what these distributions can look like at the LHC energies.
\end{abstract}

DOI: $10.1103 /$ PhysRevD.95.094010

The scenarios with extra dimensions are some of the many theoretical schemes which predict new interactions beyond the Standard Model (SM). A generic feature of these scenarios is the presence of Kaluza-Klein (KK) excitations of the graviton, which can be the first signature of such physics [1]. The corresponding effects can appear at the $\mathrm{TeV}$ energy scale, so the search for extra dimensions is one of the goals of the LHC experiments. Moreover, these scenarios, depending on the geometry of the extra dimensions, can predict relations between the fundamental Planck scale, where gravity becomes strongly coupled, and the weak scale, shedding new light on the hierarchy problem [2-7].

Several models with extra dimensions, which can be split into two main classes according to the geometry of the background space-time manifold, have been proposed. The first of them is the Arkani-Hamed-Dimopoulos-Dvali (ADD) model [2] and its variants, which postulates the existence of $n \geq 2$ large (with common size $R \gg 1 / M_{p}$, where $M_{p} \sim 10^{19} \mathrm{GeV}$ is the four-dimensional Planck scale) extra dimensions. This model assumes that all the SM particles are localized in the usual space-time, which is called the brane, while the gravity is allowed to propagate in the additional $n$-dimensional space compactified on the $n$-dimensional torus. Then the four-dimensional Planck scale is a derived scale related to the fundamental Planck scale $M_{s} \sim 1 \mathrm{TeV}$ by

$$
M_{p}^{2}=M_{s}^{n+2}(2 \pi R)^{n},
$$

thus solving the hierarchy problem. A solution of the linearized Einstein equation in $4+n$ dimensions results in the appearance of a tower of $\mathrm{KK}$ modes, which are separated in mass by $\mathcal{O}(1 / R)$ terms. After KK reduction, one has massive spin-2 KK gravitons $h_{\mu \nu}^{k}$ which interact with the SM fields via the SM energy-momentum tensor $T^{\mu \nu}$,

$$
\mathcal{L}=-\frac{\kappa}{2} \sum_{k} T^{\mu \nu}(x) h_{\mu \nu}^{k}(x)
$$

where $\kappa=\sqrt{16 \pi} / M_{p}$ and the summation runs over all KK modes.

The second scenario is the five-dimensional RandallSundrum (RS) model [3] and its variants, which implies a warped metric and in which the size of the extra dimensions should not be too large compared to the Planck length. This model is based on the solution of the Einstein equation for gravity interacting with two branes (the IR or SM brane, where our world is located at high energy, or the UV brane) in five-dimensional space-time, and the four-dimensional metric is the function of the coordinate of the fifth dimension. It is possible to explain the weakness of the gravitational interaction in comparison with the electroweak one (hierarchy problem) by the existence of warp factor in the metric. There also exist KK towers of massive spin-2 gravitons which interact with the SM fields via the effective Lagrangian [8,9],

$$
\mathcal{L}=-\frac{1}{M_{p}^{*}} T^{\mu \nu}(x) h_{\mu \nu}^{0}(x)-\frac{1}{\Lambda_{\pi}} \sum_{k} T^{\mu \nu}(x) h_{\mu \nu}^{k}(x),
$$

where $\Lambda_{\pi}$ is at the electroweak scale. The coupling of the massless graviton $h_{\mu \nu}^{0}$ is suppressed by the fourdimensional reduced Planck scale $M_{p}^{*}=M_{p} / \sqrt{8 \pi}$. The mass of the lowest graviton KK mode $m_{1}$ and $\Lambda_{\pi}$ can be considered as free parameters which completely determine 
the graviton sector of the RS model, and it is expected that $\Lambda_{\pi}<10 \mathrm{TeV}[9,10]$. The quantity $c_{0}^{*}=m_{1} / x_{1} \Lambda_{\pi}$, or rather $c_{0}=c_{0}^{*} \sqrt{8 \pi}$, is also often used as a free parameter. The masses of the $k$ th graviton KK excitation modes are given by

$$
m_{k}=m_{1} \frac{x_{k}}{x_{1}}
$$

where the $x_{k}$ are the $k$ th roots of the first-order Bessel function. So, the spectrum of KK modes is quite different from the one in the ADD scenario.

In the RS model, it is expected that the lightest massive KK graviton can have a mass $m_{1}$ of order of several hundred $\mathrm{GeV}$ (or even more). Therefore, it can be produced at the LHC and future colliders with relatively high rates. Moreover, its coupling to the SM fields is larger than the one in the ADD model, so that it can decay into observable SM particles and therefore can be detected in collider experiments. The possibility to observe the graviton signal with mass up to several $\mathrm{TeV}$ (using the $l^{+} l^{-}, \gamma \gamma, Z Z$, $W^{+} W^{-}$, and other graviton decay modes) in the protonproton collisions at the LHC was investigated [11]. The next-to-leading-order (NLO) QCD corrections to the virtual graviton production (in the $\mathrm{ADD}$ and $\mathrm{RS}$ scenarios) were calculated [10,12-14], and Collins-Soper-Sterman resummation formalism was applied [12] to take into account the soft gluon effects in the transverse momentum distributions.

Besides the KK excitation, another common characteristic feature of SM extensions involving extra dimensions is the presence of a massive scalar field (called the radion field), which has the same quantum numbers as the neutral Higgs field [15-18]. The appearance of the radion field is connected with the spin-0 metric fluctuations due to an extra space dimension. Various aspects of the decay and production properties of the radion were investigated [19-27], and it was argued [17,28] that the radion can be significantly lighter than all the other KK excitations. The interaction vertices of the radion with the SM fields are similar to those of the Higgs boson except for the anomalyenhanced interactions with gluons and photons that results in the leading role of gluon-gluon fusion production mechanism and corresponding relative enhancement in gluon and photon decay modes.

In the present paper, we apply the leading-order (LO) $k_{T}$-factorization approach $[29,30]$ to calculate the total and differential cross sections of $\mathrm{KK}$ graviton production (including its subsequent decay into the lepton or photon pair) and radion production at the LHC conditions. This approach is based on the famous Balitsky-FadinKuraev-Lipatov (BFKL) [31] or Ciafaloni-Catani-FioraniMarchesini (CCFM) [32] evolution equations and provides solid theoretical grounds for the effects of initial-state parton radiation and intrinsic parton transverse momentum. We see certain advantages in the fact that, even with the LO partonic amplitudes, one can include a large piece of higherorder corrections (namely, part of next-to-leading+ next-to-next-to-leading $+\ldots$ terms containing leading$\log 1 / x$ enhancement of cross sections due to real initialstate parton emissions), taking them into account in the form of transverse momentum dependent (TMD) parton densities. ${ }^{1}$ In this sense, the $k_{T}$-factorization approach provides an easy tool to estimate corresponding $K$-factors at high energies. The TMD parton densities in a proton additionally absorb the effects of soft gluon resummation, which regularizes the infrared divergences and makes the $k_{T}$-factorization predictions applicable even at low transverse momenta [34].

We would like to mention here an important point concerning the $k_{T}$-factorization QCD calculations beyond the LO. To perform such calculations, there are two main ingredients. The first of them is the next-to-leadinglogarithmic (NLL) corrections to the kernel of the evolution equation (for example, BFKL or CCFM), generating terms $\alpha_{s}\left(\alpha_{s} \log 1 / x\right)^{n}$. This part should be independent of the processes under consideration. The second part is the correction to the off-shell scattering amplitudes and is the source of the process dependence in the NLL corrections. For the latter, virtual and real corrections to the LO off-shell amplitudes should be investigated and put together, and the infrared finite cross sections have to be formulated before these formulas can be used in a numerical analysis, which is a special challenging task (see, for example, Refs. $[35,36])$. The NLL corrections to the BFKL equation were calculated and found to be huge [37,38], which leads to the negative value of the BFKL power which determines the rise of the gluon density at small $x$. However, the proposed approaches [39-41] considerably improve the stability of the NLL predictions for the BFKL power, giving a phenomenologically reasonable value for the latter. The NLL corrections for the CCFM equation, which is almost equivalent to BFKL in the limit of asymptotic energies and similar to the DGLAP evolution for large $x$, are still unknown. In was argued [42] that amending the leading-logarithmic evolution with different kinematical constraints should lead to reasonable QCD predictions, although still formally only to leadinglogarithmic accuracy (see also Ref. [33]). We would like to stress that the role of higher-order corrections in the $k_{T}$-factorization is very different than the ones in the collinear QCD factorization, since part of them is already included at LO level, as was mentioned above.

The $k_{T}$-factorization approach was applied to number of hard QCD processes at high energies (see, for example, Refs. [43-45] and references therein). We perform the calculations for both the ADD and RS scenarios and give

\footnotetext{
${ }^{1}$ A detailed description of the $k_{T}$-factorization approach can be found, for example, in reviews [33].
} 
special attention to the angular distributions of KK graviton decay leptons or photons since the effects of the initial gluon off-shellness are expected to be observed there. Our mail goal is to extend the applicability of the approach used by including the new processes into the $k_{T}$-factorization phenomenology and to investigate the role of gluon offshellness in these processes.

Let us start from a short review of calculation steps. First, we consider the subprocesses where dilepton or diphoton pairs are produced from off-shell gluon-gluon fusion or quark-antiquark annihilation via the virtual KK graviton exchange,

$$
\begin{aligned}
g^{*}\left(k_{1}\right)+g^{*}\left(k_{2}\right) & \rightarrow G^{*} \rightarrow l^{+}\left(p_{1}\right)+l^{-}\left(p_{2}\right), \\
q\left(k_{1}\right)+\bar{q}\left(k_{2}\right) & \rightarrow G^{*} \rightarrow l^{+}\left(p_{1}\right)+l^{-}\left(p_{2}\right), \\
g^{*}\left(k_{1}\right)+g^{*}\left(k_{2}\right) & \rightarrow G^{*} \rightarrow \gamma\left(p_{1}\right)+\gamma\left(p_{2}\right), \\
q\left(k_{1}\right)+\bar{q}\left(k_{2}\right) & \rightarrow G^{*} \rightarrow \gamma\left(p_{1}\right)+\gamma\left(p_{2}\right),
\end{aligned}
$$

where the 4-momenta of all particles are indicated in parentheses. Below, we describe the evaluation of the off-shell (transverse momentum dependent) production amplitudes, which are some of the main ingredients of the $k_{T}$-factorization approach used. In the center-of-mass frame of colliding protons, having 4-momenta $l_{1}$ and $l_{2}$, we define

$$
k_{1}=x_{1} l_{1}+k_{1 T}, \quad k_{2}=x_{2} l_{2}+k_{2 T},
$$

where $x_{1}$ and $x_{2}$ are the longitudinal momentum fractions of the protons carried by the interacting off-shell partons having transverse 4-momenta $k_{1 T}$ and $k_{2 T}$ (note that $k_{1 T}^{2}=-\mathbf{k}_{1 T}^{2} \neq 0, k_{2 T}^{2}=-\mathbf{k}_{2 T}^{2} \neq 0$ ). The relevant Feynman rules were obtained earlier [46], from which we can get the LO amplitudes of the subprocesses (5) as follows,

$$
\begin{aligned}
& \mathcal{M}\left(g^{*} g^{*} \rightarrow G^{*} \rightarrow l^{+} l^{-}\right) \\
&= \epsilon_{1 a}^{\alpha}\left(k_{1}\right) \epsilon_{2 b}^{\beta}\left(k_{2}\right) V_{\mu \nu \alpha \beta}^{a b}\left(k_{1}, k_{2}\right) \Delta_{\mu \nu \mu^{\prime} \nu^{\prime}}\left(k_{1}+k_{2}\right) \\
& \times \bar{u}_{r_{1}}\left(p_{1}\right) \Gamma^{\mu^{\prime} \nu^{\prime}}\left(p_{1}, p_{2}\right) v_{r_{2}}\left(p_{2}\right), \\
& \mathcal{M}\left(q \bar{q} \rightarrow G^{*} \rightarrow l^{+} l^{-}\right) \\
&= \bar{v}_{s_{1}}\left(k_{2}\right) \Gamma^{\mu \nu}\left(k_{1}, k_{2}\right) u_{s_{2}}\left(k_{1}\right) \Delta_{\mu \nu \mu^{\prime} \nu^{\prime}}\left(k_{1}+k_{2}\right) \\
& \times \bar{u}_{r_{1}}\left(p_{1}\right) \Gamma^{\mu^{\prime} \nu^{\prime}}\left(p_{1}, p_{2}\right) v_{r_{2}}\left(p_{2}\right), \\
& \mathcal{M}\left(g^{*} g^{*} \rightarrow G^{*} \rightarrow \gamma \gamma\right) \\
&= \epsilon_{1 a}^{\alpha}\left(k_{1}\right) \epsilon_{2 b}^{\beta}\left(k_{2}\right) V_{\mu \nu \alpha \beta}^{a b}\left(k_{1}, k_{2}\right) \Delta_{\mu \nu \mu^{\prime} \nu^{\prime}}\left(k_{1}+k_{2}\right) \\
& \times V^{\mu^{\prime} \nu^{\prime} \alpha^{\prime} \beta^{\prime}}\left(p_{1}, p_{2}\right) e_{1 \alpha^{\prime}}\left(p_{1}\right) e_{2 \beta^{\prime}}\left(p_{2}\right), \\
& \mathcal{M}\left(q \bar{q} \rightarrow G^{*} \rightarrow \gamma \gamma\right) \\
&=\bar{v}_{s_{1}}\left(k_{2}\right) \Gamma^{\mu \nu}\left(k_{1}, k_{2}\right) u_{s_{2}}\left(k_{1}\right) \Delta_{\mu \nu \mu^{\prime} \nu^{\prime}}\left(k_{1}+k_{2}\right) \\
& \quad \times V^{\mu^{\prime} \nu^{\prime} \alpha^{\prime} \beta^{\prime}}\left(p_{1}, p_{2}\right) e_{1 \alpha^{\prime}}\left(p_{1}\right) e_{2 \beta^{\prime}}\left(p_{2}\right),
\end{aligned}
$$

where $a$ and $b$ are the eight-fold color indices and $\epsilon_{\mu}^{a}(k)$ and $e_{\mu}(p)$ are the polarization vectors of initial off-shell gluons and produced photons, respectively. In the RS model, the interaction vertices of gluons $V_{\mu \nu \alpha \beta}^{a b}\left(k_{1}, k_{2}\right)$ and fermions $\Gamma_{\mu \nu}\left(k_{1}, k_{2}\right)$ with the graviton can be written as

$$
\begin{aligned}
V_{\mu \nu \alpha \beta}^{a b}= & -i \frac{1}{\Lambda_{\pi}} \delta^{a b}\left[\left(k_{1} \cdot k_{2}\right) C_{\mu \nu \alpha \beta}\right. \\
& \left.+D_{\mu \nu \alpha \beta}\left(k_{1}, k_{2}\right)+E_{\mu \nu \alpha \beta}\left(k_{1}, k_{2}\right)\right], \\
\Gamma_{\mu \nu}\left(k_{1}, k_{2}\right)= & -i \frac{1}{4 \Lambda_{\pi}}\left[\gamma_{\mu}\left(k_{1}-k_{2}\right)_{\nu}+\gamma_{\nu}\left(k_{1}-k_{2}\right)_{\mu}\right. \\
& \left.-2 \eta_{\mu \nu}\left(\hat{k}_{1}-\hat{k}_{2}-2 m_{f}\right)\right],
\end{aligned}
$$

where $m_{f}$ is the fermion mass and $\eta_{\mu \nu}$ is the Minkowski metrics tensor. The tensors appearing in (11) are defined as [46]

$$
\begin{gathered}
C_{\mu \nu \alpha \beta}=\eta_{\mu \alpha} \eta_{\nu \beta}+\eta_{\mu \beta} \eta_{\nu \alpha}-\eta_{\mu \nu} \eta_{\alpha \beta}, \\
D_{\mu \nu \alpha \beta}\left(k_{1}, k_{2}\right)=\eta_{\mu \nu} k_{1 \beta} k_{2 \alpha}-\left[\eta_{\mu \beta} k_{1 \nu} k_{2 \alpha}+\eta_{\mu \alpha} k_{1 \beta} k_{2 \nu}\right. \\
\left.-\eta_{\alpha \beta} k_{1 \mu} k_{2 \nu}+(\mu \leftrightarrow \nu)\right], \\
E_{\mu \nu \alpha \beta}\left(k_{1}, k_{2}\right)=\eta_{\mu \nu}\left(k_{1 \alpha} k_{1 \beta}+k_{2 \alpha} k_{2 \beta}+k_{1 \alpha} k_{2 \beta}\right) \\
-\left[\eta_{\nu \beta} k_{1 \mu} k_{1 \alpha}+\eta_{\nu \alpha} k_{2 \mu} k_{2 \beta}+(\mu \leftrightarrow \nu)\right],
\end{gathered}
$$

and for the graviton propagator, one has

$$
\Delta_{\mu \nu \alpha \beta}(p)=\frac{(i / 2) B_{\mu \nu \alpha \beta}(p)}{p^{2}-m^{2}+i \Gamma m},
$$

where $m$ is the graviton mass, $\Gamma$ is its full decay width, and polarization sum $B_{\mu \nu \alpha \beta}(p)$ takes the following form:

$$
B_{\mu \nu \alpha \beta}(p)=\eta_{\mu \alpha} \eta_{\nu \beta}+\eta_{\mu \beta} \eta_{\nu \alpha}-\frac{2}{3} \eta_{\mu \nu} \eta_{\alpha \beta}+\cdots
$$

The dots represent terms proportional to the graviton momentum $p$ which give a vanishing contribution due to the gauge invariance. Below, we neglect the virtualities of the initial quarks in the production amplitudes (8) and (10) compared to the large scale (but not in the kinematics), so that their spin density matrix is taken in the usual form

$$
\sum_{r, s} u_{r}(p) \bar{u}_{s}(p)=\hat{p}+m_{q},
$$

where $m_{q}$ is the quark mass. According to the $k_{T}$-factorization prescription [29,30], the summation over the polarizations of incoming off-shell gluons is carried with

$$
\sum \epsilon^{\mu}(k) \epsilon^{* \nu}(k)=\frac{\mathbf{k}_{T}^{\mu} \mathbf{k}_{T}^{\nu}}{\mathbf{k}_{T}^{2}}
$$


thus avoiding diagrams involving ghosts. In the limit of collinear QCD factorization, when $\mathbf{k}_{T}^{2} \rightarrow 0$, this expression converges to the ordinary one after averaging on the azimuthal angle.

The partial decay widths of the KK graviton to SM particles via the Lagrangian (3) are known [46]. So, the partial decay width of the KK graviton to massless gauge bosons $\Gamma_{V_{0} V_{0}}$, massive gauge bosons $\Gamma_{V V}$, fermions $\Gamma_{f f}$, and the Higgs boson $\Gamma_{H H}$ are

$$
\begin{gathered}
\Gamma_{V_{0} V_{0}}=\frac{C m^{3}}{80 \pi \Lambda_{\pi}^{2}} \\
\Gamma_{V V}=\delta \frac{m^{3}}{40 \pi \Lambda_{\pi}^{2}}\left(1-\frac{4 m_{V}^{2}}{m^{2}}\right)^{1 / 2}\left(\frac{13}{12}+\frac{14 m_{V}^{2}}{39 m^{2}}+\frac{4 m_{V}^{4}}{13 m^{4}}\right), \\
\Gamma_{f f}=\delta \frac{C m^{3}}{160 \pi \Lambda_{\pi}^{2}}\left(1-\frac{4 m_{f}^{2}}{m^{2}}\right)^{3 / 2}\left(1+\frac{8 m_{f}^{2}}{3 m^{2}}\right) \\
\Gamma_{H H}=\frac{m^{3}}{480 \pi \Lambda_{\pi}^{2}}\left(1-\frac{4 m_{H}^{2}}{m^{2}}\right)^{5 / 2}
\end{gathered}
$$

where $m_{V}, m_{f}$, and $m_{H}$ are the gauge bosons, fermions, and Higgs boson masses; $C$ is the color factor $\left(C=N_{c}^{2}-1\right.$ for gluons, $C=N_{c}$ for quarks, and $C=1$ for colorless particles, where $N_{c}$ is the number of colors); $\delta=1 / 2$ for self-conjugate particles; and $\delta=1$ for other particles.

To extend the formulas above to the ADD model, one has to replace $1 / \Lambda_{\pi} \rightarrow \kappa / 2$ and perform the summation over a high multiplicity of KK modes lying below the UV cutoff scale (given by $M_{s}$ ). This summation compensates the suppression of each KK mode coupling to the SM particles by Planck mass and gives rise to a substantial effective coupling strength. Following Refs. [46,47], we have to replace the graviton propagator by the effective one,

$$
\mathcal{P}_{\text {eff }}=\sum_{k} \frac{i}{p^{2}-m_{k}^{2}+i \Gamma_{k} m_{k}},
$$

where $k$ sums over all KK towers below the $M_{s}$ scale. As was already mentioned above, in the ADD model, the mass separation between two adjacent KK modes is a $\mathcal{O}(1 / R)$, so that KK modes become quasicontinuous and the summation in (24) can be done by defining KK-state density [46,47]. For $n$ number of extra dimensions, the $\mathcal{P}_{\text {eff }}$ is given by

$$
\mathcal{P}_{\text {eff }}=\frac{16 \pi \hat{s}^{n / 2-1}}{\kappa^{2} \Gamma(n / 2) M_{s}^{n+2}}\left[\pi+2 i I\left(\frac{M_{s}}{\sqrt{\hat{s}}}\right)\right],
$$

where $\hat{s}=p^{2}$ is the invariant mass of the produced dilepton or diphoton pair, and the function $I(\tau)$ reads

$$
I(\tau)= \begin{cases}-\sum_{k=1}^{n / 2-1} \frac{\tau^{2 k}}{2 k}-\frac{1}{2} \ln \left(\tau^{2}-1\right), & n=\text { even } \\ -\sum_{k=1}^{(n-1) / 2} \frac{\tau^{2 k-1}}{2 k-1}+\frac{1}{2} \ln \left(\frac{\tau+1}{\tau-1}\right), & n=\text { odd }\end{cases}
$$

The real part of (25) comes from the summation over all resonant contributions below $M_{s}$, and the imaginary part is the summed contributions coming from all the nonresonant states. Further calculations are straightforward and follow the standard QCD Feynman rules. The evaluation of the traces was performed using the algebraic manipulation system FORM [48]. The obtained analytical expressions are too lengthy to be presented here, but they are available from the authors upon request. ${ }^{2}$

The amplitude of radion production in the off-shell gluon-gluon fusion can be easily obtained using the effective vertex $[20,26]$,

$$
\begin{aligned}
T^{\mu \nu}\left(k_{1}, k_{2}\right)= & i \delta^{a b} \frac{\alpha_{s}}{2 \pi} \frac{1}{\Lambda_{r}}\left[b_{\mathrm{QCD}}+F\left(\frac{4 m_{t}^{2}}{m_{r}^{2}}\right)\right] \\
& \times\left(k_{2}^{\mu} k_{1}^{\nu}-\left(k_{1} \cdot k_{2}\right) g^{\mu \nu}\right),
\end{aligned}
$$

where $a$ and $b$ are the eight-fold color indices, $m_{t}$ and $m_{r}$ are the top quark and radion masses, the radion coupling constant $\Lambda_{r}$ is supposed to be of order of the electroweak scale, $b_{\mathrm{QCD}}=7$, and $F$ is the known function (see, for example, Refs. [20,26]). The further evaluation is rather straightforward. We only mention that the summation on the initial off-shell gluon polarizations was done using (19).

To calculate the graviton production cross section in the $k_{T}$-factorization approach, one has to convolute the relevant off-shell partonic cross section and the TMD parton densities in a proton. Our master formulas for gluon-gluon fusion and quark-antiquark annihilation read

$$
\begin{aligned}
\sigma= & \int \frac{|\overline{\mathcal{M}}|^{2}}{16 \pi\left(x_{1} x_{2} s\right)^{2}} f_{g}\left(x_{1}, \mathbf{k}_{1 T}^{2}, \mu^{2}\right) \\
& \times f_{g}\left(x_{2}, \mathbf{k}_{2 T}^{2}, \mu^{2}\right) d \mathbf{p}_{1 T}^{2} d \mathbf{k}_{1 T}^{2} d \mathbf{k}_{2 T}^{2} d y_{1} d y_{2} \frac{d \phi_{1}}{2 \pi} \frac{d \phi_{2}}{2 \pi}, \\
\sigma= & \sum_{q} \int \frac{|\overline{\mathcal{M}}|^{2}}{16 \pi\left(x_{1} x_{2} s\right)^{2}} f_{q}\left(x_{1}, \mathbf{k}_{1 T}^{2}, \mu^{2}\right) \\
& \times f_{\bar{q}}\left(x_{2}, \mathbf{k}_{2 T}^{2}, \mu^{2}\right) d \mathbf{p}_{1 T}^{2} d \mathbf{k}_{1 T}^{2} d \mathbf{k}_{2 T}^{2} d y_{1} d y_{2} \frac{d \phi_{1}}{2 \pi} \frac{d \phi_{2}}{2 \pi},
\end{aligned}
$$

where $f_{q}\left(x, \mathbf{k}_{T}^{2}, \mu^{2}\right)$ and $f_{g}\left(x, \mathbf{k}_{T}^{2}, \mu^{2}\right)$ are the TMD quark and gluon densities in a proton; $|\overline{\mathcal{M}}|^{2}$ is the corresponding

\footnotetext{
${ }^{2}$ lipatov@theory.sinp.msu.ru.
} 

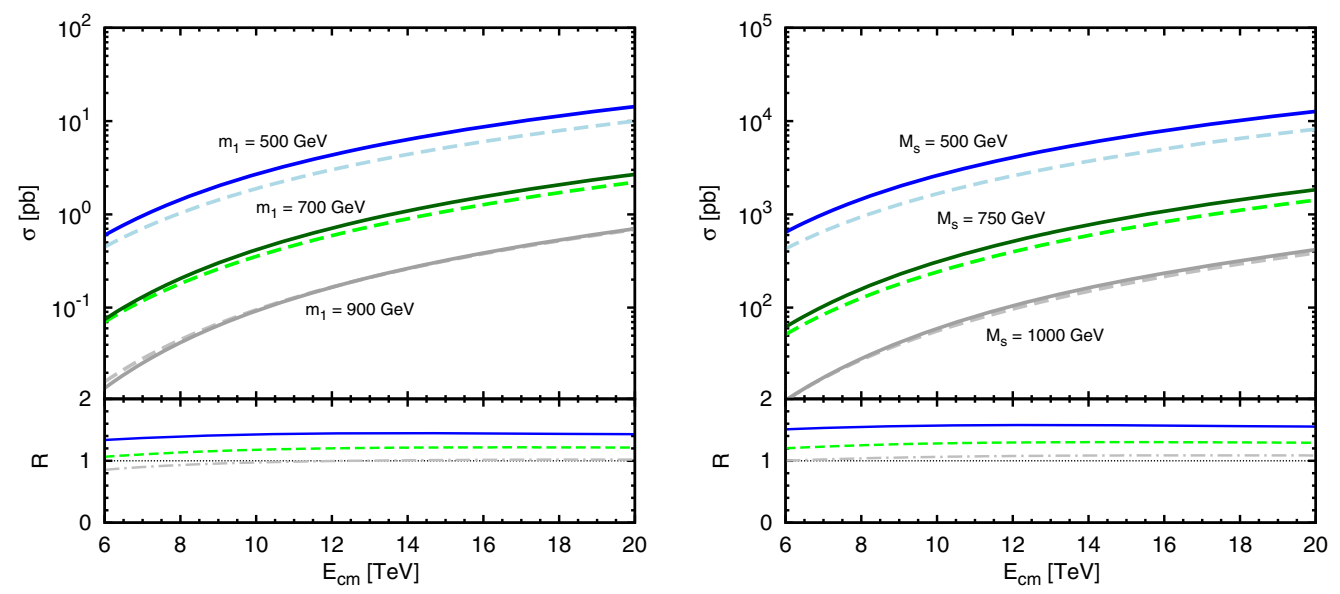

FIG. 1. The cross sections of KK graviton production with its subsequent dilepton decay calculated as a function of the total center-ofmass energy in the RS model with $c_{0}=0.01$ (left panel) and the ADD model with $n=3$ (right panel). The solid and dashed curves correspond to the $k_{T}$-factorization and LO pQCD predictions, respectively. The ratios of these predictions at $m_{1}=500,700$, and $900 \mathrm{GeV}$ for the RS model and $M_{s}=500,750$, and $1000 \mathrm{GeV}$ for the ADD model are shown below by the solid, dashed, and dashdotted curves.

production amplitude squared (and averaged over initial state and summed over final states), $\mathbf{p}_{1 T} ; y_{1}$ and $y_{2}$ are the transverse momentum and rapidities of produced particles; and $\sqrt{s}$ is the $p p$ center-of-mass energy. A similar expression can be obtained for the radion production. In the case of two-photon decay of the KK state, one has to include an extra factor $1 / 2$ when integrating over full phase space due to the identity of the final-state photons. If we average (27) and (28) over $\phi_{1}$ and $\phi_{2}$ and take the limit $\mathbf{k}_{1 T}^{2} \rightarrow 0$ and $\mathbf{k}_{2 T}^{2} \rightarrow 0$, then we recover relevant expressions of LO collinear QCD approximation.

Concerning the TMD quark and gluon densities, we use the CCFM-evolved gluon [49] and valence quark distributions [50] as given by the A0 set, which are commonly recognized and widely applied in the phenomenological applications. ${ }^{3}$ The CCFM evolution equation is the most suitable tool for our present study because it smoothly interpolates between the small- $x$ BFKL gluon dynamics and the conventional DGLAP one. The corresponding input parameters were fitted from the best description of the proton structure function $F_{2}\left(x, Q^{2}\right)$. The TMD sea quark density is calculated in the approximation, where the sea quarks occur in the last gluon splitting [52] using the TMD gluon-to-quark splitting function [53].

Numerically, the renormalization and factorization scales $\mu_{R}$ and $\mu_{F}$ were set to be equal to $\mu_{R}^{2}=\mu_{F}^{2}=\hat{s}+\mathbf{Q}_{T}^{2}$ as a default choice, where $\mathbf{Q}_{T}$ is the transverse momentum of the initial gluon or quark pair. The choice of $\mu_{F}^{2}$ is connected with the CCFM evolution [49]. Other essential

\footnotetext{
${ }^{3}$ More recently, another set of the CCFM-evolved parton distributions in a proton, the JH'2013 one, was presented [51]. However, the relevant TMD gluon density does not reproduce the behavior of standard gluon distributions at large $x$. Therefore, we do not use it in our consideration below.
}

parameters were taken as follows. Unless mentioned otherwise, we chose the parameters $c_{0}=0.01$ for the RS model and $n=3$ for the ADD model and performed calculations for different $m_{1}$ and $M_{s}$ values, respectively. Everywhere, we used LO formula for the strong coupling constant $\alpha_{s}\left(\mu^{2}\right)$ with $n_{f}=4$ massless quark flavors and $\Lambda_{\mathrm{QCD}}=200 \mathrm{MeV}$, so that $\alpha_{s}\left(M_{Z}^{2}\right)=0.1232$. The multidimensional integration was performed by means of a Monte Carlo technique, using the routine vEGAS [54].

We now are in a position to present our numerical results. In Figs. 1 and 2, we plot the total cross sections of KK graviton production and its subsequent decays into the dilepton and diphoton pair calculated as functions of center-of-mass energy for several values of parameters $m_{1}$ and $M_{s}$ (in the RS and ADD models, respectively). Here, we compare the $k_{T}$-factorization predictions and the ones obtained in the collinear QCD factorization (at the LO level). For the conventional parton densities in a proton, we adopted the LO Martin-Stirling-Thorn-Watt (MSTW'2008) set [55]. One can see that at relatively large values of $m_{1}$ or $M_{s}$ (namely, about of $1 \mathrm{TeV}$ ) the $k_{T}$-factorization predictions practically coincide with the LO perturbative QCD (pQCD) ones, and the difference between them occurs only at smaller $m_{1}$ or $M_{s}$ values. It can be easily understood if we consider the scaling variable $z=m_{1} / \sqrt{s}$ ( or $M_{s} / \sqrt{s}$ ), which can serve as an estimation of momentum fraction $x$ the interacting particles. At the LHC energies, this variable is about $z \sim 0.1$ when $m_{1}$ or $M_{s}$ is about $1 \mathrm{TeV}$ and going down to $z \sim 5 \times 10^{-2}$ for lower $m_{1}$ or $M_{s}$ values (about $500 \mathrm{GeV}$ ). The corresponding variable $R$, which can be defined as a ratio between the $k_{T}$-factorization and LO QCD predictions, changes from $R \sim 1$ to $R \sim 1.4-1.6$ (see Figs. 1 and 2). As was mentioned above, the $k_{T}$-factorization approach (supplemented with the BFKL or CCFM gluon dynamics) effectively includes a 

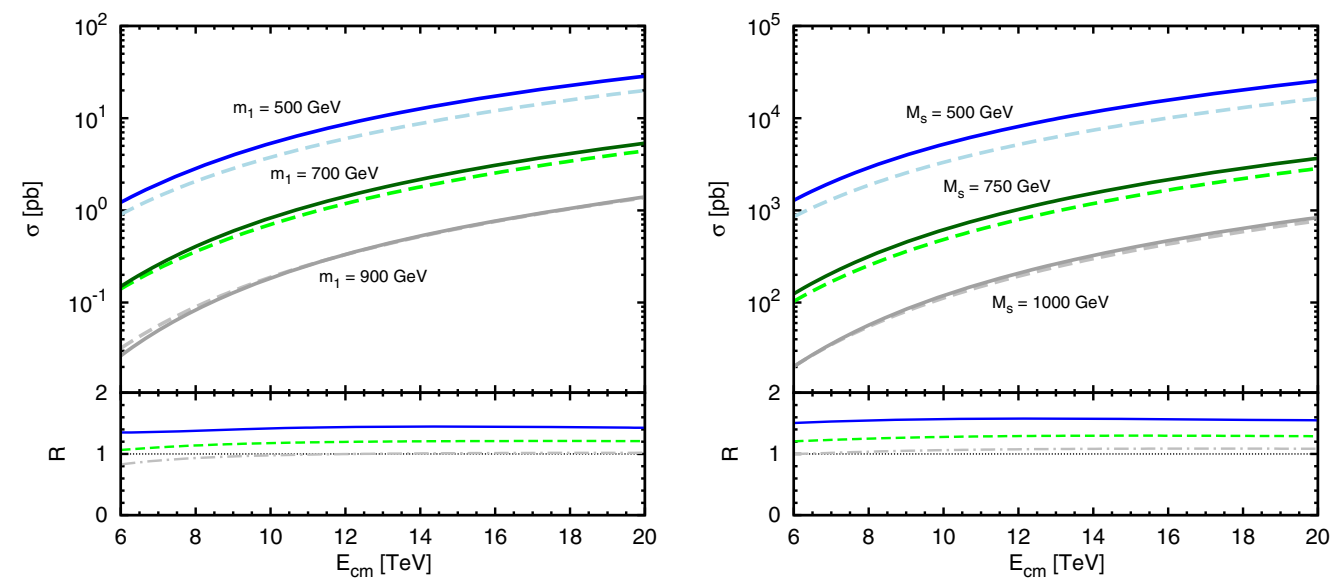

FIG. 2. The cross sections of KK graviton production with its subsequent diphoton decay calculated as a function of the total center-ofmass energy in the RS model with $c_{0}=0.01$ (left panel) and the ADD model with $n=3$ (right panel). Notation of all curves is the same as in Fig. 1.

part of $\mathrm{NLO}+\mathrm{NNLO}+\ldots$ terms containing leading $-\log 1 / x$ enhancement of cross sections, so that this ratio $R$ reflects the role of such terms in the total NLO pQCD corrections. According to the estimates [10,12-14], the latter (so-called $K$-factor) is about $K \sim 1.7-1.8$ at the LHC conditions. So, one can conclude that the $k_{T}$-factorization predictions lie between the LO and NLO pQCD ones in the considered kinematical region and the contribution from the leading $\log 1 / x$ terms to the total NLO corrections is significant. ${ }^{4}$

In the considered energy range, the main contributions to the total production cross sections come from the gluongluon fusion subprocesses. They give about $94.8 \%, 91.1 \%$, and $87.1 \%$ contributions at $m_{1}=500,700$, and $900 \mathrm{GeV}$, respectively. The quark-antiquark initiated subprocesses are suppressed due to large gluon flux at the LHC.

Now, we turn to discuss the uncertainties of our calculations connected with the hard scale and/or the TMD parton distributions in a proton. To estimate the uncertainty coming from the renormalization scale $\mu_{R}$, we used the CCFM set $\mathrm{A} 0+$ and $\mathrm{A} 0-$ instead of the default $\mathrm{A} 0$ one. These two sets represent a variation of the scale used in the strong coupling constant in the off-shell matrix element (see Ref. [49]). The $\mathrm{A} 0+$ stands for a variation of $2 \mu_{R}$, while set $\mathrm{A} 0-$ reflects $\mu_{R} / 2$. In all analyses studied here, we observe a deviation of roughly $30 \%$ around the default predictions, as is demonstrated in Fig. 3. In the collinear QCD factorization, the LO cross sections vary by about $18 \%$ when the hard scales range between $500 \mathrm{GeV}$ and $4 \mathrm{TeV}$ [12-14]. The scale dependence of the NLO cross sections is much smaller than that of the LO ones; it varies by $9 \%$ approximately [12-14]. The significant scale dependence of the $k_{T}$-factorization

\footnotetext{
${ }^{4}$ At very high energies or, alternatively, in the small- $x$ region, the $\log 1 / x$-enhanced terms, corresponding to the real initial-state parton emissions, give the main contribution to the production cross section.
}

predictions is not surprising since the tree-level LO hard scattering amplitudes are involved.

Since the main subprocess contributing to the $\mathrm{KK}$ graviton production is the off-shell gluon-gluon fusion, the calculated cross sections should strongly depend on the TMD gluon densities used. At the moment, there is a large variety of the latter. In some cases, the differences between them arise because different approaches employ similar TMD concepts but are adapted to specific physical applications, which complicates efforts to compare different parametrizations. Most of the proposed TMD gluon densities are collected in the TMDLIB package [56], which is a $\mathrm{C}++$ library that provides a framework and an interface to the different parametrizations. To investigate the sensitivity of our predictions to the TMD gluon densities, we repeated the calculations using the ones from the B0 set [49]. Similar to $\mathrm{A} 0$, the $\mathrm{B} 0$ gluon density is also obtained from the numerical solution of the CCFM evolution equation, where the input parameters were fitted to the $F_{2}$ data with some different (compared to the $\mathrm{A} 0$ ones) values of the soft cut and width of the intrinsic $k_{T}$ distribution. The results of our calculations are shown in Fig. 3. We find that the B0 predictions are below the A0 ones and close to the lower bound of the $\mathrm{A} 0$ scale uncertainties band (but still inside the latter). However, we point out that B0 gluon density underestimates the LHC data on the $b$-quark and $B$-meson production [45]. The main reason to use the A0 gluon density as a default choice in our numerical calculations is that this distribution reproduces well the number of experimental data on the different processes (like the production of charmed and beauty quarks, $b$-hadrons, Drell-Yan lepton pairs, and Higgs bosons) studied at the HERA, Tevatron, and LHC conditions. ${ }^{5}$ So, we consider the latter as one of the most realistic TMD gluon distribution functions, and all the

\footnotetext{
${ }^{5}$ See, for example, Refs, [43-45] and references therein.
} 



FIG. 3. The cross sections of KK graviton production with its subsequent dilepton (left panel) and diphoton (right panel) decays calculated as a function of the total center-of-mass energy in the RS model with $c_{0}=0.01$ and $m_{1}=500 \mathrm{GeV}$. The solid curves represent the predictions obtained with the A0 TMD parton densities at the default scale, whereas the shaded band represents the scale uncertainties of these calculations, as is described in the text. The dashed lines correspond to the predictions obtained with the B0 TMD parton distributions.

results below are obtained with the default A0 density. In the collinear QCD factorization and the RS scenario, the uncertainties connected with the parton distributions are about $5 \%$ at $m_{1} \sim 500 \mathrm{GeV}$ and about $15 \%$ at $m_{1} \sim 1.5 \mathrm{TeV}$ [12-14]. In the considered $m_{1}$ range, the PDF uncertainties in the LO and NLO cross sections are about the same.

As it is known, the effects connected with the noncollinear parton dynamics can manifest themselves in the different angular correlations between the final-state particles [33]. A first (and rather trivial) example is the distributions in the azimuthal angle difference $\Delta \phi$ between the produced leptons or photons. In the collinear LO pQCD approximation, this distribution must be simply a delta function $\delta(\Delta \phi-\pi)$, since the produced leptons or photons are back to back in the transverse momentum plane. Taking into account the nonvanishing parton transverse momentum leads to a violation of this back-to-back kinematics in the $k_{T}$-factorization approach even at LO, while in the collinear QCD factorization, such a violation occurs at NLO level only. The same can be noted for the transverse momentum distributions of produced dilepton or diphoton pairs because the latter is determined by the transverse momenta of incoming partons. These effects are illustrated in Fig. 4, where we show the $p_{T}^{l l}$ and $\Delta \phi^{l l}$ distributions calculated in the RS scenario at $c_{0}=0.01$ and $\sqrt{s}=14 \mathrm{TeV}$. As one can see, the $k_{T}$-factorization predictions are finite at any $p_{T}^{l l}$ and $\Delta \phi^{l l}$ values. In the collinear QCD factorization, to make the predictions at low $p_{T}^{l l} \ll M$ (where $M$ is the invariant mass of
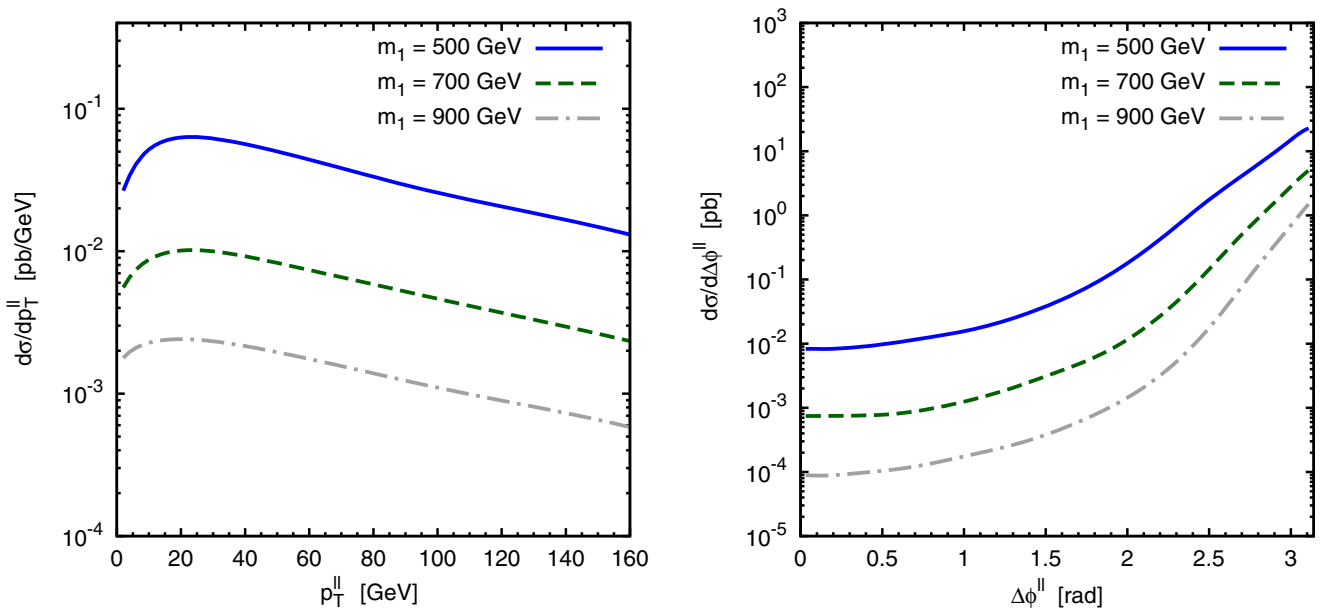

FIG. 4. The distributions on the KK decay dilepton transverse momentum $p_{T}^{l l}$ (left panel) and azimuthal angle difference $\Delta \phi^{l l}$ (right panel) between the 4-momenta of these leptons calculated at $\sqrt{s}=14 \mathrm{TeV}$ in the RS model with $c_{0}=0.01$. The solid, dashed, and dash-dotted curves correspond to $m_{1}=500,700$, and $900 \mathrm{GeV}$, respectively. 

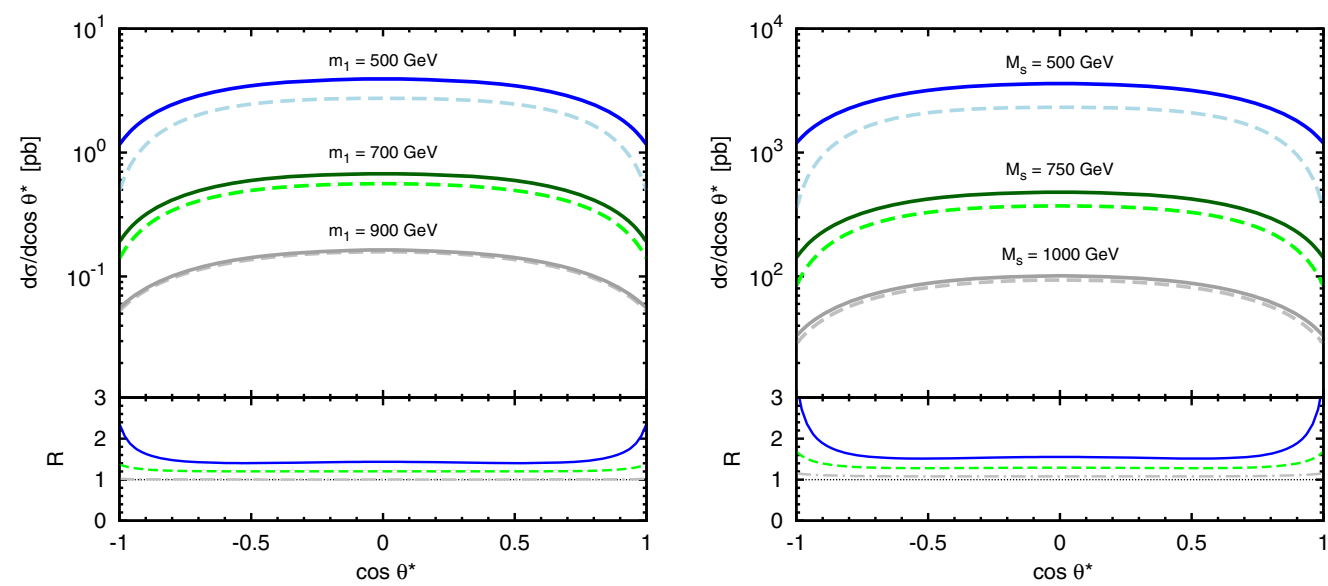

FIG. 5. The angular distributions $d \sigma / d \cos \theta^{*}$ of the KK graviton decay lepton pair calculated in the RS model with $c_{0}=0.01$ (left panel) and the ADD model with $n=3$ (right panel) at $\sqrt{s}=14 \mathrm{TeV}$. Notation of all curves is the same as in Fig. 1.

dilepton pair), one should use a special soft gluon resummation technique since perturbative QCD calculations at fixed-order diverge at small dilepton transverse momenta with terms proportional to $\ln M / p_{T}^{l l}$ appearing due to soft and collinear gluon emission. However, as was shown [34], the soft gluon resummation formulas are the result of the approximate treatment of the solution of the CCFM evolution equation, implemented in our calculations.

Another example is the distributions in the scattering angle $\theta^{*}$ of KK decay leptons or photons in the KK graviton center-of-mass frame. At LO, the on-shell gluon-gluon fusion processes, which dominate at the LHC energies, behave as $1-\cos ^{4} \theta^{*}$ and $1+6 \cos ^{2} \theta^{*}+\cos ^{4} \theta^{*}$ for dilepton and diphoton decay modes, respectively (see, for example, Refs. $[46,47])$. In the $k_{T}$-factorization approach, taking into account the initial gluon off-shellness can result in the deviations from these simple forms, as is demonstrated in Figs. 5 and 6. For the dilepton KK decay mode, we find that the ratio $R$ is more or less flat at $\left|\cos \theta^{*}\right| \leq 0.8-0.9$, so that the difference in shape between the $k_{T}$-factorization and collinear LO pQCD predictions occurs at $\left|\cos \theta^{*}\right| \geq 0.9$ and becomes more clearly pronounced when the $z$ variable decreases. In the diphoton decay mode, this effect occurs if $\left|\cos \theta^{*}\right|$ is close to zero. In fact, the reason for such shape differences is the presence of additional contributions from the longitudinal polarizations of initial off-shell gluons, which are absent in the collinear QCD calculations. To investigate the influence of offshell gluon longitudinal polarization in more detail, we repeated these calculations, performing the summation over the gluons polarizations explicitly, choosing appropriate expressions for gluon polarization 4-vectors. Our results are shown in Fig. 7, where the contributions from different polarizations are displayed separately. As an illustration, we consider here the gluon-gluon fusion only for both dilepton and diphoton decay modes and set $M_{s}=500 \mathrm{GeV}$ (in the ADD model with $n=3$ ) to enlarge the visible effect. One can see that the contributions from pure transverse gluon polarizations generally follow the collinear QCD predictions, whereas the contributions from the
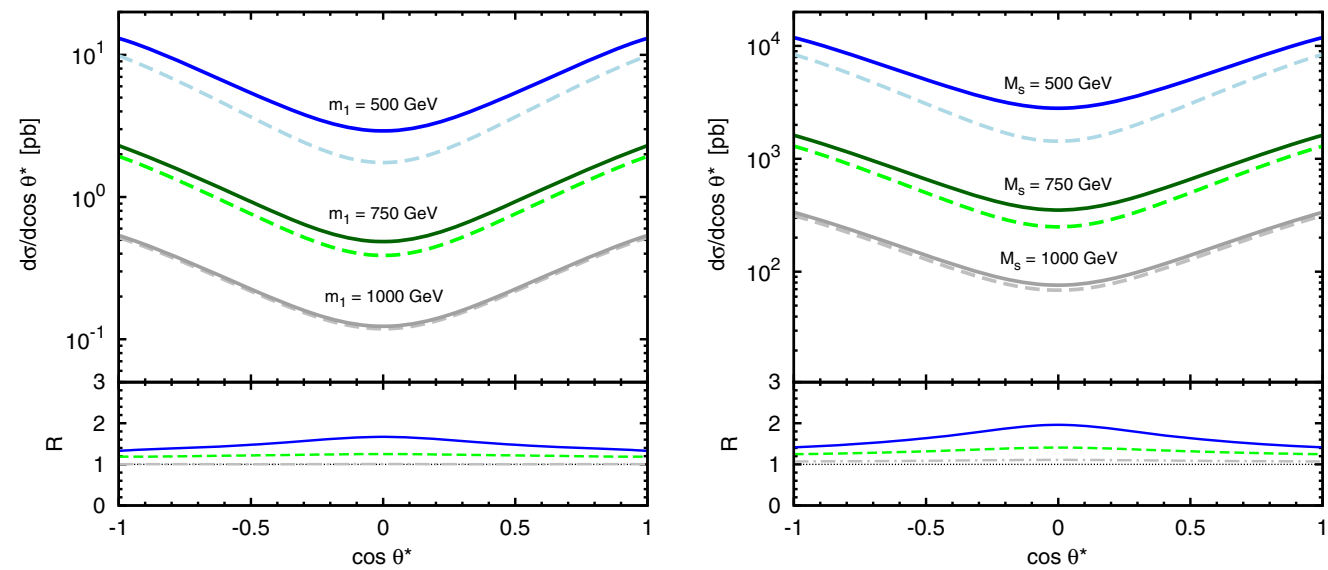

FIG. 6. The angular distributions $d \sigma / d \cos \theta^{*}$ of the KK graviton decay photon pair calculated in the RS model with $c_{0}=0.01$ (left panel) and the ADD model with $n=3$ (right panel) at $\sqrt{s}=14 \mathrm{TeV}$. Notation of all curves is the same as in Fig. 1. 

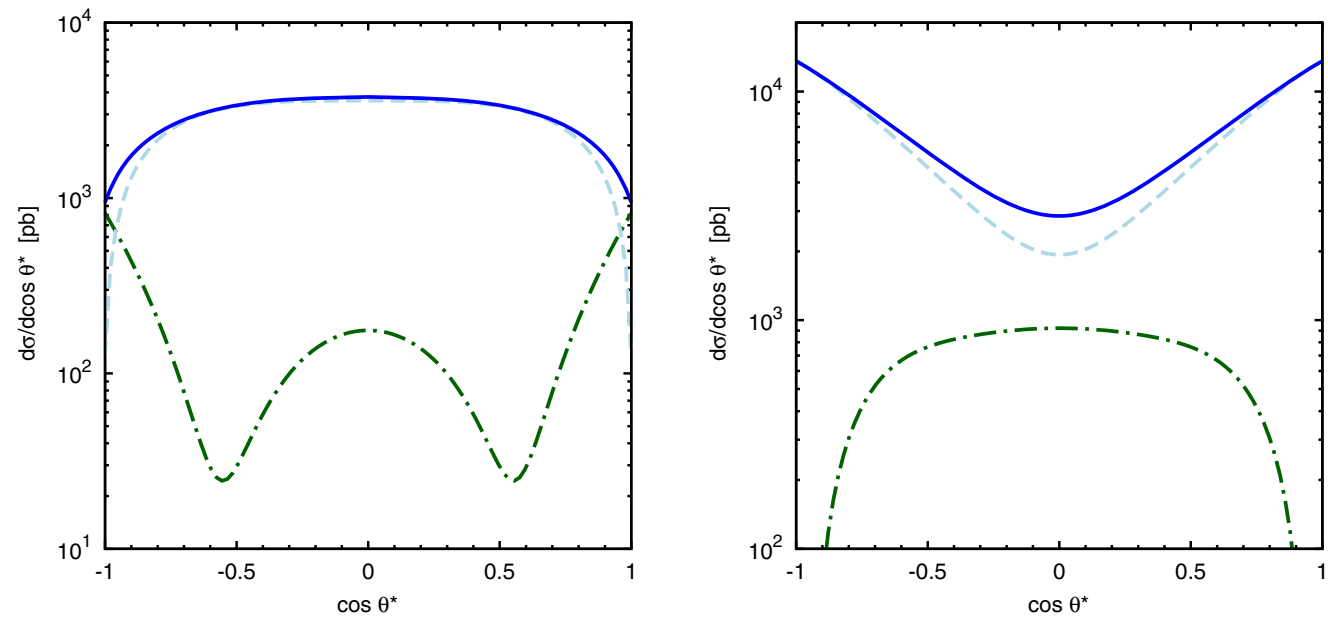

FIG. 7. The contributions from transversal and longitudinal off-shell gluon polarizations to the $d \sigma / d \cos \theta^{*}$ distributions for dilepton (left panel) and diphoton (right panel) KK graviton decay modes calculated in the ADD model with $n=3, M_{s}=500 \mathrm{GeV}$ and $\sqrt{s}=14 \mathrm{TeV}$. The dashed and dash-dotted curves correspond to the transversal and longitudinal components, respectively. The solid curves represent their sum.

longitudinal off-shell gluon polarization have different behavior in $\cos \theta^{*}$, which leads to the observed deviations from the LO pQCD results. The latter, of course, is embodied in the different polarization of produced particles and, in principle, can be observed experimentally. The same effect was pointed out in the heavy quarkonia production at high energies (see, for example, Ref. [57]). As was demonstrated, the fraction of longitudinally polarized quarkonia increases in the $k_{T}$-factorization approach in comparison with the collinear QCD predictions, which is a direct consequence of the enhancement of the longitudinal component in the polarization vectors of initial off-shell gluons. As one can see from Figs. 5 and 6, at the large values of scaling variable $z$, where the small- $x$ region is not probed, the pointed effect becomes negligible.

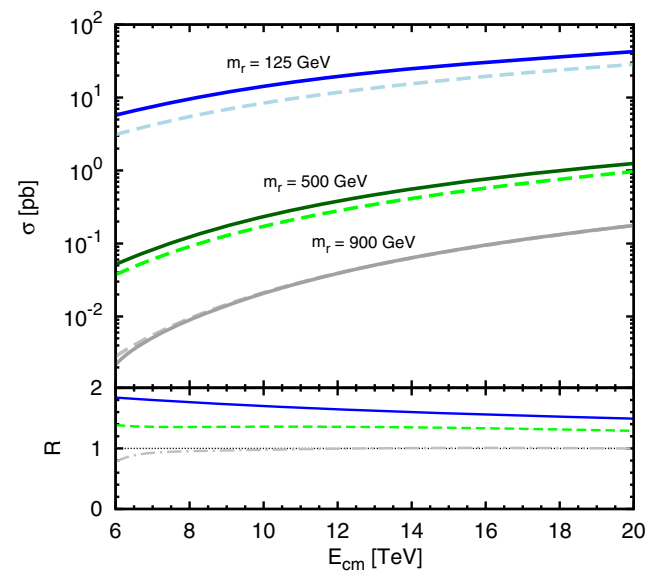

Now, we turn to the radion production. Our predictions are shown in Fig. 8, where we plot the total cross sections of radion production calculated as functions of center-of-mass energy and transverse momentum distributions calculated for several values of radion mass. According to estimates [26], the latter can either be about $125 \mathrm{GeV}$ or be close to the $\mathrm{TeV}$ range, depending on the radion coupling constant. Numerically, we set $m_{r}=125,500$, or $900 \mathrm{GeV}$ with $\Lambda_{r}=$ $3 \mathrm{TeV}$ [26]. As one can see, our predictions follow the same trend as previous ones for graviton production: at relatively large radion mass, $m_{r} \sim 1 \mathrm{TeV}$, there is practically no difference between the $k_{T}$-factorization and LO pQCD calculations. The difference occurs at smaller values of radion mass, where essentially the small- $x$ region is probed. The predicted transverse momentum distributions,

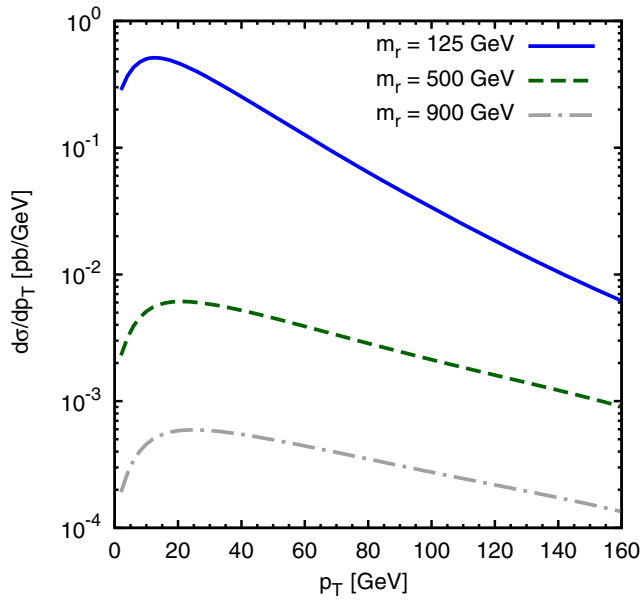

FIG. 8. The total cross sections (left panel) and transverse momentum distributions (right panel) of radion production calculated for several values of radion mass $m_{r}$ at $\Lambda_{r}=3 \mathrm{TeV}$. Notation of all curves in the left panel is the same as in Fig. 1. The transverse momentum distributions are calculated at $\sqrt{s}=14 \mathrm{TeV}$. 
shown in Fig. 8, are finite and determined by the TMD gluon densities in a proton due to $2 \rightarrow 1$ subprocess kinematics.

Finally, we would like to note that recent experimental searches performed by the CMS [58,59] and ATLAS $[60,61]$ collaborations put the lower bound on scale $M_{s}$ quite high, $M_{s}>2-3 \mathrm{TeV}$, thus eliminating the visible small- $x$ effects in the ADD model (which appear at lowest $M_{s}$ values, as we can see in Figs. 1 and 2). The similar situation holds for the RS scenario. So, the CMS report [62] constrains the parameters of the RS model using very recent $13 \mathrm{TeV}$ data. In this analysis, the $\mathrm{KK}$ graviton masses $m_{1}$ below $1.35 \mathrm{TeV}$ are excluded for $c_{0}^{*}=0.01$. For $c_{0}^{*}=0.1$ and $c_{0}^{*}=0.2$, the graviton masses below 3.1 and $3.8 \mathrm{TeV}$ are excluded also [62]. However, even with such a large $M_{s}$ scale or $m_{1}$ mass, the small- $x$ effects can appear in future collider experiments, like CERN Future Circular Collider, where total energy of about $\sqrt{s}=100 \mathrm{TeV}$ is expected to be reached. Additionally, the recent observation $[63,64]$ of a $750 \mathrm{GeV}$ resonance in the diphoton spectrum at the LHC caused a number of interpretations as a RS graviton (see, for example, Refs. [65-68] and references therein). In particular, the value of coupling scale $\Lambda_{\pi} \simeq$ $60_{-10}^{+12} \mathrm{TeV}$, that corresponds to approximately $0.01<c_{0}<$ 0.02 , was obtained [65] from the best fit to the combined ATLAS and CMS data $[63,64]$. But this resonance observation was not confirmed later.

To conclude, we applied the $k_{T}$-factorization approach to investigate the Kaluza-Klein graviton production (including its subsequent decay into a dilepton and/or diphoton pair) and radion production at the LHC. We considered the Arkani-Hamed-Dimopoulos-Dvali and Randall-Sundrum scenarios and derived the corresponding amplitudes for spin-2 KK-state production and spin-0 radion production in the off-shell gluon-gluon fusion. In the case of subleading quark-induced subprocesses, we neglected the initial quarks transverse momenta in the production amplitudes but kept the exact off-shell kinematics. We used the transverse momentum dependent quark and gluon densities in a proton obtained from the CCFM evolution equation, which resums the leading logarithmic small- $x$ corrections to the production cross section. It is important that the CCFM equation covers both small- $x$ and large- $x$ regions. We point out that we did not try to give better predictions for KK production than the fixed-order $\mathrm{PQCD}$ calculations. Our main goal was to extend the applicability of the $k_{T}$-factorization approach by including the new processes in the consideration and to investigate the role of gluon offshellness in these processes. We found that the small- $x$ effects (which impact the overall normalization of calculated cross sections or polarization of the final states) in the KK graviton production at the LHC appear if the lightest graviton mass is of order of $700 \mathrm{GeV}$ or below (in the RS model). A similar conclusion was reached for the radion production. In the ADD scenario, the small- $x$ effects appear at $M_{s} \leq 750 \mathrm{GeV}$. We gave some examples of how these effects, connected with the off-shell parton kinematics or additional longitudinal polarization of initial off-shell gluons, can manifest themselves in the different angular distributions of produced particles.

\section{ACKNOWLEDGMENTS}

We thank E. E. Boos, M. N. Smolyakov, V. E. Bunichev, S. P. Baranov, H. Jung, and F. Hautmann for very useful discussions and important remarks. In the initial stage this work was supported in by Grant No. 14-12-00363 of the Russian Science Foundation. Also this work was supported in part by RFBR Grant No. 16-32-00176-mol-a, President of Russian Federation Grant No. NS-7989.2016.2, and by the DESY Directorate in the framework of the MoscowDESY project on Monte Carlo implementations for HERA-LHC.
[1] M. J. Duff, B. E. W. Nilsson, and C. N. Pope, Phys. Rep. 130, 1 (1986).

[2] N. Arkani-Hamed, S. Dimopoulos, and G. R. Dvali, Phys. Lett. B 429, 263 (1998).

[3] L. Randall and R. Sundrum, Phys. Rev. Lett. 83, 3370 (1999).

[4] J. D. Lykken, Phys. Rev. D 54, R3693 (1996).

[5] E. Witten, Nucl. Phys. B471, 135 (1996).

[6] P. Horava and E. Witten, Nucl. Phys. B460, 506 (1996).

[7] I. Antoniadis, Phys. Lett. B 246, 377 (1990).

[8] J. L. Hewett, Phys. Rev. Lett. 82, 4765 (1999).
[9] H. Davoudiasl, J. L. Hewett, and T. G. Rizzo, Phys. Rev. Lett. 84, 2080 (2000).

[10] P. Mathews, V. Ravindran, and K. Sridhar, J. High Energy Phys. 10 (2005) 031.

[11] E. E. Boos, V.E. Bunichev, M. N. Smolyakov, and I. P. Volobuev, Phys. Rev. D 79, 104013 (2009).

[12] Q. Li, C. S. Li, and L. L. Yang, Phys. Rev. D 74, 056002 (2006).

[13] M. C. Kumar, P. Mathews, V. Ravindran, and A. Tripathi, Nucl. Phys. B818, 28 (2009).

[14] J. Gao, C. S. Li, B. H. Li, C. P. Yuan, and H. X. Zhu, Phys. Rev. D 82, 014020 (2010). 
[15] W. D. Goldberger and M. B. Wise, Phys. Rev. Lett. 83, 4922 (1999).

[16] O. DeWolfe, D. Z. Freedman, S. S. Gubser, and A. Karch, Phys. Rev. D 62, 046008 (2000).

[17] E. E. Boos, Y. S. Mikhailov, M. N. Smolyakov, and I. P. Volobuev, Nucl. Phys. B717, 19 (2005).

[18] E. E. Boos, Y. S. Mikhailov, M. N. Smolyakov, and I. P. Volobuev, Mod. Phys. Lett. A 21, 1431 (2006).

[19] G. F. Giudice, R. Rattazzi, and J. D. Wells, Nucl. Phys. B595, 250 (2001).

[20] K.-M. Cheung, Phys. Rev. D 63, 056007 (2001).

[21] M. Chaichian, A. Datta, K. Huitu, and Z.-H. Yu, Phys. Lett. B 524, 161 (2002).

[22] T. G. Rizzo, J. High Energy Phys. 06 (2002) 056.

[23] D. Dominici, B. Grzadkowski, J. F. Gunion, and M. Toharia, Nucl. Phys. B671, 243 (2003).

[24] J. F. Gunion, M. Toharia, and J. D. Wells, Phys. Lett. B 585, 295 (2004).

[25] E. E. Boos, S. Keizerov, E. Rakhmetov, and K. Svirina, Phys. Rev. D 90, 095026 (2014).

[26] E. E. Boos, V. E. Bunichev, M. A. Perfilov, M. N. Smolyakov, and I. P. Volobuev, Phys. Rev. D 92, 095010 (2015).

[27] E. E. Boos, S. Keizerov, E. Rakhmetov, and K. Svirina, Phys. Rev. D 94, 024047 (2016).

[28] C. Csaki, M. L. Graesser, and G. D. Kribs, Phys. Rev. D 63, 065002 (2001).

[29] L. V. Gribov, E. M. Levin, and M. G. Ryskin, Phys. Rep. 100, 1 (1983); E. M. Levin, M. G. Ryskin, Yu. M. Shabelsky, and A. G. Shuvaev, Sov. J. Nucl. Phys. 53, 657 (1991).

[30] S. Catani, M. Ciafoloni, and F. Hautmann, Nucl. Phys. B366, 135 (1991); J. C. Collins and R. K. Ellis, Nucl. Phys. B360, 3 (1991).

[31] E. A. Kuraev, L. N. Lipatov, and V. S. Fadin, Sov. Phys. JETP 44, 443 (1976); E. A. Kuraev, L. N. Lipatov, and V. S. Fadin, Sov. Phys. JETP 45, 199 (1977); I. I. Balitsky and L. N. Lipatov, Sov. J. Nucl. Phys. 28, 822 (1978).

[32] M. Ciafaloni, Nucl. Phys. B296, 49 (1988); S. Catani, F. Fiorani, and G. Marchesini, Phys. Lett. B 234, 339 (1990); Nucl. Phys. B336, 18 (1990); G. Marchesini, Nucl. Phys. B445, 49 (1995).

[33] B. Andersson et al. (Small- $x$ Collaboration), Eur. Phys. J. C 25, 77 (2002); J. Andersen et al. (Small-x Collaboration), Eur. Phys. J. C 35, 67 (2004); J. Andersen et al. (Small- $x$ Collaboration), Eur. Phys. J. C 48, 53 (2006).

[34] A. Gawron and J. Kwiecinski, Phys. Rev. D 70, 014003 (2004).

[35] J. Bartels, S. Gieseke, and C. F. Qiao, Phys. Rev. D 63, 056014 (2001).
[36] J. Bartels, S. Gieseke, and A. Kyrieleis, Phys. Rev. D 65, 014006 (2001).

[37] V. S. Fadin and L. N. Lipatov, Phys. Lett. B 429, 127 (1998).

[38] M. Ciafaloni and G. Camici, Phys. Lett. B 430, 349 (1998).

[39] G. Salam, J. High Energy Phys. 07 (1998) 019.

[40] M. Ciafaloni and D. Colferai, Phys. Lett. B 452, 372 (1999).

[41] M. Ciafaloni, D. Colferai, and G. P. Salam, Phys. Rev. D 60, 114036 (1999).

[42] H. Jung, S. P. Baranov, M. Deak, A. Grebenyuk, F. Hautmann, M. Hentschinski, A. Knutsson, M. Kraemer, K. Kutak, A. V. Lipatov, and N. P. Zotov, Eur. Phys. J. C 70, 1237 (2010).

[43] A. V. Lipatov, M. A. Malyshev, and N. P. Zotov, Phys. Lett. B 735, 79 (2014).

[44] S. P. Baranov, A. V. Lipatov, and N. P. Zotov, Phys. Rev. D 89, 094025 (2014).

[45] H. Jung, M. Krämer, A. V. Lipatov, and N. P. Zotov, Phys. Rev. D 85, 034035 (2012).

[46] T. Han, J. D. Lykken, and R. J. Zhang, Phys. Rev. D 59, 105006 (1999).

[47] R. Basu and T. Mandal, Adv. High Energy Phys. 2013, 652714 (2013).

[48] J. A. M. Vermaseren, Report No. NIKHEF-00-023, 2000.

[49] H. Jung, arXiv:hep-ph/0411287.

[50] M. Deak, H. Jung, and K. Kutak, arXiv:0807.2403.

[51] F. Hautmann and H. Jung, Nucl. Phys. B883, 1 (2014).

[52] F. Hautmann, M. Hentschinski, and H. Jung, Nucl. Phys. B865, 54 (2012).

[53] S. Catani and F. Hautmann, Nucl. Phys. B427, 475 (1994).

[54] G. P. Lepage, J. Comput. Phys. 27, 192 (1978).

[55] A. D. Martin, W. J. Stirling, R. S. Thorne, and G. Watt, Eur. Phys. J. C 63, 189 (2009).

[56] http://tmd.hepforge.org.

[57] S. P. Baranov, Phys. Lett. B 428, 377 (1998).

[58] CMS Collaboration, Phys. Lett. B 711, 15 (2012).

[59] CMS Collaboration, Phys. Rev. Lett. 108, 111801 (2012).

[60] ATLAS Collaboration, Phys. Rev. D 87, 015010 (2013).

[61] ATLAS Collaboration, New J. Phys. 15, 043007 (2013).

[62] CMS Collaboration, Tech. Rep. CMS-PAS-EXO-15-004, 2015.

[63] ATLAS Collaboration, J. High Energy Phys. 09 (2016) 001.

[64] CMS Collaboration, Phys. Rev. Lett. 117, 051802 (2016).

[65] M. T. Arun and P. Saha, arXiv:1512.06335.

[66] S. B. Giddings and H. Zhang, Phys. Rev. D 93, 115002 (2016).

[67] B. M. Dillon and V. Sanz, arXiv:1603.09550.

[68] B. M. Dillon, C. Han, H. M. Lee, and M. Park, arXiv:1606.07171. 